\title{
The Anesthetic Biobank of Cerebrospinal fluid; storing CSF for future neuroscientific research
}

\author{
Atmosoerodjo S.D. ${ }^{1}$, Absalom A.R. ${ }^{1}$
}

1. University Medical Center Groningen, University of Groningen, Groningen, The Netherlands.

university of

groningen

\section{Background and goal}

- More knowledge of the neurobiology of the cerebrospinal fluid (CSF) is needed for a better understanding of the pathophysiology of degenerative brain diseases and for biomarker development

- There is a need for CSF samples from healthier patients from a wider age span

- In collaboration with colleagues from our neurology and clinical chemistry departments, we have set up the Anesthetic Biobank of Cerebrospinal fluid $(A B C)$ to facilitate the banking of CSF from patients without clinical suspicions of cognitive or neurological problems, who are undergoing planned spinal anesthesia

\section{Materials and methods}

- All patients $>18$ years scheduled for elective (inpatient or outpatient) surgery under spinal anesthesia are invited to participate

- After puncture of the sub-arachnoid space and prior to administering spinal anesthesia, $10 \mathrm{ml}$ of CSF is aspirated with five $2 \mathrm{ml}$ syringes (this takes approximately 3 minutes). The maximum block height and any subsequent episodes of PDPH are recorded

- The CSF and blood is immediately transferred on melting ice and sent to the laboratory. The first $2 \mathrm{ml}$ of CSF and $10 \mathrm{ml}$ of blood is used for cell count, glucose, albumin and protein analysis. The remaining $8 \mathrm{ml} \mathrm{CSF}$ and $10 \mathrm{ml}$ blood is centrifuged and stored at $-70^{\circ} \mathrm{C}$ in $0.4 \mathrm{ml}$ aliquots

- Data regarding patient characteristics, medical history, surgery, spinal anesthesia and the postoperative period (length of stay, complications, clinical delirium scores) are stored in a database

Figure 1. Flowchart of inclusion procedure / data handling.

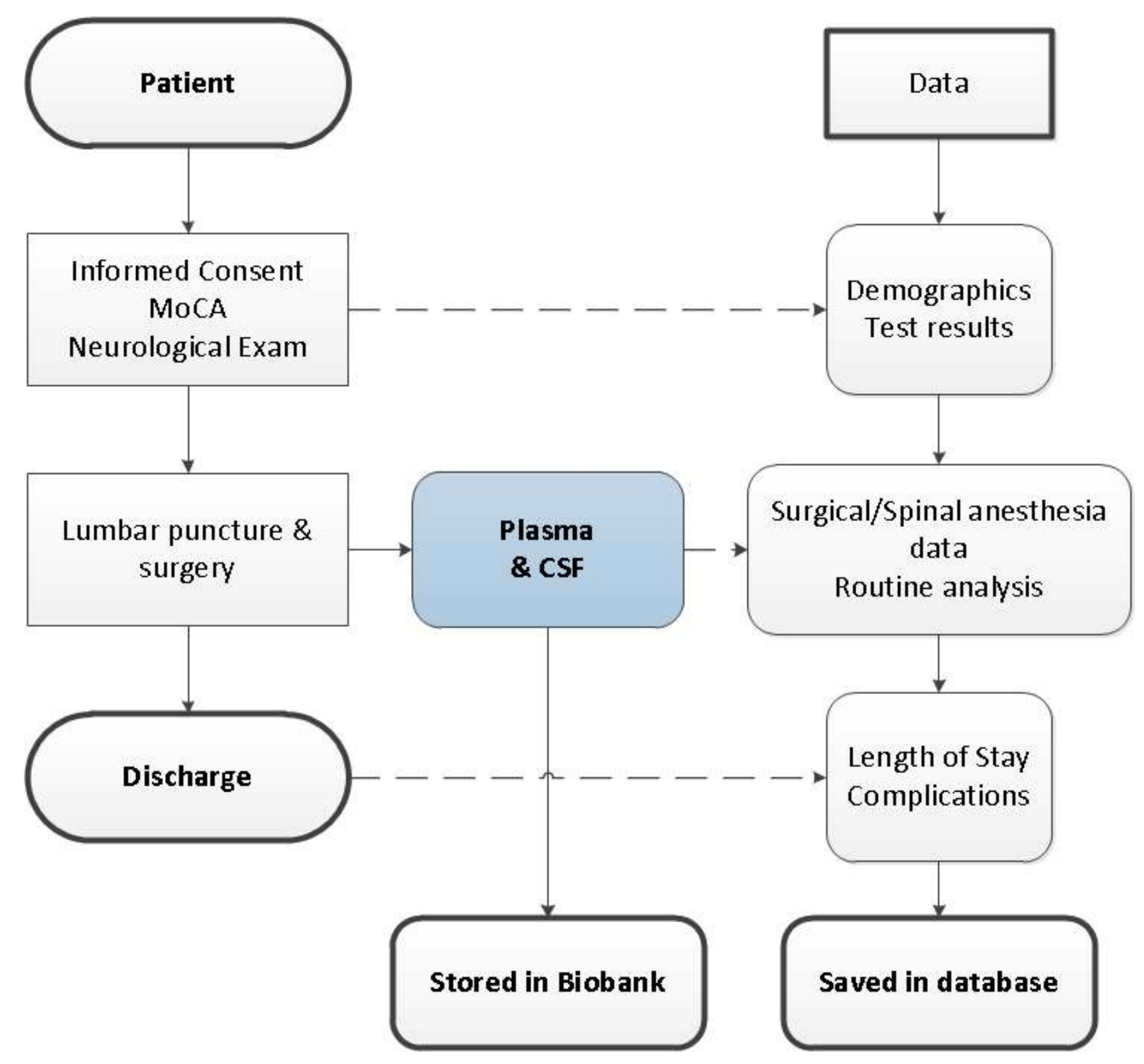

\section{Current status \& future developments}

- After 4 months of inclusion 100 patients have participated

- The Biobank is expected to contain 200 CSF and blood samples across all age groups before 2018

- One episode of PDPH occurred (27 years, female). After discharge home the patient showed features of a low CSF pressure headache. Her symptoms settled spontaneously over a few days without any interventions

- Five patients had a vaso-vagal reaction during CSF aspiration. In 2 cases the aspiration attempt was stopped prematurely. In the other 3 cases a full $10 \mathrm{ml}$ was obtained. In all 5 cases, the symptoms improved with administration of $10 \mathrm{mg}$ ephedrine and/or $0,5 \mathrm{mg}$ atropine

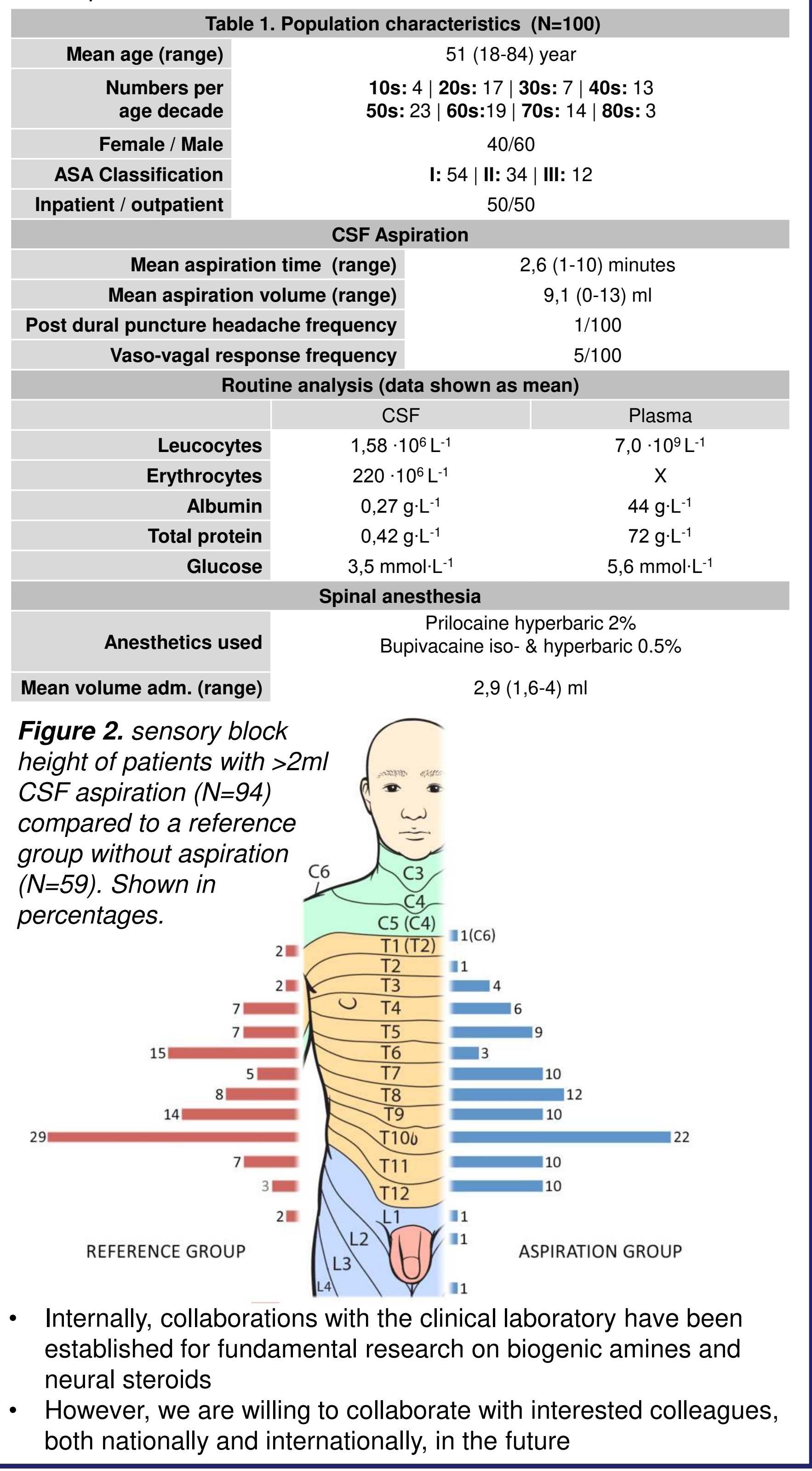

